\title{
Effects of Exercise Training on Functional Capacity and Quality of Life in a Patient with Late-Onset Pompe Disease Receiving Enzyme Replacement Therapy
}

\author{
Rosângela M. Silva*, Carmen S.C. Mendes, Carolina C. Aranda, Marco A. Curiati, Maret H. Rand, \\ Sandra O. Kyosen, Edna T. Sakata, Sueli Canossa, Renata B. Oliveira, Ana M. Martins \\ Centro de Referências em Erros Inatos do Metabolismo (CREIM), Department of Pediatrics, Universidade \\ Federal de São Paulo (Unifesp), São Paulo, Brazil
}

\section{BACKGROUND}

Pompe disease (PD) causes a progressive muscular weakness and impairment of patients' mobility. Many patients develop intolerance to exercise due to reduced activity and consequent decline in muscle function. Exercise training prevents physical deconditioning and muscle wasting. Enzyme replacement therapy (ERT) was recently introduced, and has modified the course of the disease. Other therapeutic interventions, such as exercise training, have been proposed as possible countermeasures for muscle impairment in patients with late-onset PD. We report the results of a 12-week home exercise training program on functional capacity and quality of life in a 20 -year-old male patient with PD receiving ERT for 2 years.

\section{MATERIALS AND METHODS}

The patient was assessed before and after the training period, following protocols: (1) 6-minute walk test (6MWT), according to ATS criteria; and (2) quality of life (QoL) related to health status was evaluated by the 36-item Short Form Questionnaire (SF36). The home-based exercise training consisted of two parts: aerobic and stretching exercises for the major muscle groups before and after training. Aerobic exercise consisted of the initial guidance: 30 minutes walking on flat ground or cycling in a sitting position. The exercise started slowly,

\footnotetext{
*Correspondence to: Rosângela M. Silva, Centro de Referências em Erros Inatos do Metabolismo (CREIM), Department of

Pediatrics, Universidade Federal de Sao Paulo - Unifesp, Coronel Lisboa, 957; Vila Clementino, São Paulo 04020-041, Brazil, Tel.: +55011 5081 9620; Fax: +55 0115081 9624. E-mail: rosangela_ epm@yahoo.com.br.
}

allowing periods of rest, followed by a gradual increase in exercise intensity, reaching aerobic levels of $60 \%$ of maximum heart rate, $3-5 \times /$ week at home and bi-weekly under the supervision of a physiotherapist at our service (Reference Center for Inborn Errors of Metabolism [CREIM/Unifesp]). The patient received a booklet explaining the prescribed exercises.

\section{RESULTS}

At baseline, the SF-36 had a worst score in the domains: physical functioning $($ score $=25)$, pain ( score $=$ 30 ), and functional capacity (score $=40$ ), and better score in the areas: emotional ( score $=100)$ and mental health (score $=84)$. After 12 weeks of aerobic training, performance at the 6MWT improved $(326 \mathrm{~m}$ before vs. $372 \mathrm{~m}$ after), and the scores of the domains physical functioning $($ score $=100)$, pain (score $=$ $100)$, and functional capacity ( score $=45$ ) of the questionnaire SF-36.

\section{CONCLUSIONS}

Home-based aerobic exercise training seems to have a positive effect on functional capacity and QoL, reflected in the domains: functional capacity, physical aspects, and pain, which assess the impact of physical health and pain in performing activities of daily living. The sedentary lifestyle of this patient might be worsening the deleterious effects on the skeletal muscles, so an individualized exercise program can be considered to prevent and treat these effects.

\section{FINANCIAL SUPPORT}

Institute of Genetics and Inborn Errors of Metabolism (IGEIM). 